\title{
Traveling wave solutions for $(3+1)$ dimensional equations arising in fluid mechanics
}

\begin{abstract}
In this note, traveling wave solutions for $(3+1)$ dimensional fluid models of incompressible flow are considered. The governing partial differential equations of two models are reduced to ordinary differential equation by employing wave parameter and exact solutions are obtained. It is shown that these fluid models allow $3+1 \mathrm{di}-$ mensional solutions amongst each other. The methodology used in this work is independent of symmetric consideration and other restrictive assumption. Finally, a set of example of boundary condition is discussed for the couple stress fluid. It is observed that velocity profile strongly depends upon couple stress parameter.
\end{abstract}

Keywords: Three dimensional flow; traveling wave; exact solution; couple stress fluid

DOI 10.1515/nleng-2014-0010

Received March 12, 2014; accepted October 31, 2014.

\section{Introduction}

During the past few decades, the flow of Newtonain and non-Newtonain fluids have been analyzed and considered by various investigators $[6,8-10,15-17]$. The understanding of flow characteristics of these fluids is of significant importance, because they play a substantial role in industry, engineering and in diverse areas of biorheology, geophysics, chemical and petroleum industries. In order to study flow behavior of these kinds of non-Newtonian lubricants, many microcontinuum theories have been generated [2, 3, 21]. Among these theories, the Stokes [21] microcontinuum theory is the simplest generalization of the classical theory of fluids which allows for polar effects such as the presence of couple stresses and body couples in a continuous medium. When the bearings are subjected to reciprocating loads, the lubricants may become con-

\footnotetext{
*Corresponding Author: Najeeb Alam Khan: Department of Mathematical Sciences, University of Karachi, Karachi 75270, Pakistan, E-mail: njbalam@yahoo.com

Hassam Khan : Department of Mathematical Sciences, University of Karachi, Karachi 75270, Pakistan
}

taminated with dirt and metalparticles then the lubricant behaves as a fluid suspension. The classical Newtonian theory will not predict the accurate flow behavior of fluid suspensions especially when the clearance in the bearing is comparable with average size of the lubricant additives. The consideration of couple stress in addition to the classical Cauchy stress has led to the recent development of theories of fluid microcontinua. This new branch of fluid mechanics has attracted a growing interest during recent years mainly because it possesses the mechanism to describe such rheologically complex fluids, for which the Navier-Stokes theory is inadequate. It is well known that the theory of couple stress fluid is a generalization of the viscous fluid theory with couple stresses and body couples. In a viscous medium, this fluid contains rigid and random-oreinted particles. The couple stress fluid features anti-symmetric tensor and viscous fluid theory fails to explain the charecteristics of such fluids. This model has been proposed to take account of particle-size effects and is significant for scientific and engineering applications of pumping fluids such as complex lubricants. Liquid crystals, colloidal fluids, liquid containing long-chain molecules as polymer suspensions, animal and human blood, and lubrication are some examples of couple stress fluids. Srivastava [22] considered the peristaltic transport of a couple-stress fluid. Farooq et al. [7] investigated the heat transfer analysis of the couple stress fluid between two parallel plates with variable viscosity. Rani et al. [19] presented the couple stress fluid over an infinite vertical cylinder. Effects of Hall and ion-slip on couple stress fluid between the parallel disks is examined by [24]. Adesanya et al. [1] studied the MHD couple stress pulsatile flow between parallel plates. Effects of Newtonian heating on the magnetohydrodynmaics (MHD) three-dimensional flow past a stretching surface are analyzed by [9]. El-Dabe et al. [5] discussed the effects of couple stresses on pulstile hydromagnetic Poiseille flow.

It is established fact that these flow equations are nonlinear partial differential equations and few exact solutions in two dimensions of these equations are reported by various investigators. To obtain exact solutions of these equations are a current topic of research. Therefore investigators are concerned in obtaining exact solutions of 
such equations by employing various methods.The mathematical setting governing the non-linear fluids present more non-linear and higher order equation than the Naveir Stokes equation. Therefore, any research focusing on the exact solution always excites the researcher and deserve a special attention. Undoubtly, exact solutions are valid on the whole domain of the definition whereas the numerical solution are just valid at chosen point on the domain of definition.

The study of the traveling wave solution of non-linear equations led to the comprehension of nonlinear physical phenomena [4] in mathematical biology, chemical kinetics, and fluid mechanics [12, 13].

For the present paper, the method is as follows: In section 2, we put forward the main steps of the problems. A theoretical development has also been given in that section. While section 3 discusses the application for the flow of $(3+1)$ dimensional Newtonain and couple stress fluid respectively. And concluding remarks are synthesized in section 4.

\section{Summary of the method}

The method under consideration can be written for the given system of coupled PDEs

$$
\begin{gathered}
E_{1}\left(u_{x}, v_{y}, w_{z}\right)=0 \\
E_{2}\left(u, v, w, p_{x}, u_{t}, u_{x}, u_{y}, u_{z}, u_{x x}, \ldots, v_{t}, v_{x}, v_{y}, v_{z},\right. \\
\left.v_{x x}, \ldots, w_{t}, w_{x}, w_{y}, w_{z}, w_{x x}\right)=0 \\
E_{3}\left(u, v, w, p_{y}, u_{t}, u_{x}, u_{y}, u_{z}, u_{x x} \ldots, v_{t}, v_{x}, v_{y}, v_{z},\right. \\
\left.v_{x x}, \ldots, w_{t}, w_{x}, w_{y}, w_{z}, w_{x x}\right)=0 \\
E_{4}\left(u, v, w, p_{z}, u_{t}, u_{x}, u_{y}, u_{z}, u_{x x}, \ldots, v_{x x}, v_{y y}, \ldots,\right. \\
\left.w_{t}, w_{x}, w_{y}, w_{z}, w_{x x}, \ldots\right)=0
\end{gathered}
$$

We look for the following traveling wave solutions

$$
\begin{aligned}
u(x, y, z, t) & =u(\xi), v(x, y, z, t)=v(\xi), w(x, y, z, t) \\
& =w(\xi), p(x, y, z, t)=p(\xi)
\end{aligned}
$$

where $\xi=m x+n y+c z+d t$. Then system (1)-(4) are reduced to ordinary differential equations

$$
\begin{gathered}
F_{1}\left(u^{\prime}, v^{\prime}, w^{\prime}\right)=0 \\
F_{2}\left(u, v, w, p^{\prime}, u^{\prime}, u^{\prime \prime}, u^{\prime \prime \prime}, \ldots v^{\prime}, v^{\prime \prime}, v^{\prime \prime \prime}, \ldots w, w^{\prime}, w^{\prime \prime}\right)=0
\end{gathered}
$$

$$
F_{3}\left(u, v, w, p^{\prime}, u^{\prime}, u^{\prime \prime}, u^{\prime \prime \prime} \ldots v^{\prime}, v^{\prime \prime}, v^{\prime \prime \prime}, \ldots w, w^{\prime}, w^{\prime \prime}\right)=0
$$

$$
F_{4}\left(u, v, w, p^{\prime}, u^{\prime \prime}, v^{\prime \prime \prime}, \ldots w, w^{\prime}, w^{\prime \prime} \ldots\right)=0
$$

where $m, n, c$, and $t$ are the constants, prime denotes the derivatives with respect to $x$. The solution of the system of the ordinary differential equation can be obtained by eliminating pressure $p$ from equations of momentum making use of the equation of the continuity. The obtained equation may integrate easily for variables $u, v$ and $w$. Let us apply this methodology for two different flow equations.

\section{Application $1(3+1$ Newtonain fluid)}

The equations of incompressible three dimensional unsteady Newtonain fluid are governed by the system

$$
\nabla \cdot V=0
$$

$$
\rho\left(V_{t}+(V . \nabla) V\right)=-\nabla p-\mu(\nabla \times \nabla \times V)
$$

Here, $V$ is the velocity, $p$ is the fluid pressure function, $\mu$ is the constant viscosity, $\rho$ is the density.

In present paper, we shall consider an unsteady Newtonain three dimensional fluid by taking

$V=(u(x, y, z, t), v(x, y, z, t), w(x, y, z, t)), p=p(x, y, z, t)$.

So, flow equations can be written

$$
u_{x}+v_{y}+w_{z}=0
$$

$$
\begin{aligned}
& u_{t}+u u_{x}+v u_{y}+w u_{z}=-\frac{p_{x}}{\rho}+\frac{\mu}{\rho}\left(u_{x x}+u_{y y}+u_{z z}\right) \\
& v_{t}+u v_{x}+v v_{y}+w v_{z}=-\frac{p_{y}}{\rho}+\frac{\mu}{\rho}\left(v_{x x}+v_{y y}+v_{z z}\right)
\end{aligned}
$$

$$
w_{t}+u w_{x}+v w_{y}+w w_{z}=-\frac{p_{z}}{\rho}+\frac{\mu}{\rho}\left(w_{x x}+w_{y y}+w_{z z}\right)
$$

$\frac{\mu}{\rho}$ is equal to $v$. On substituting the representation of the solution into (11)-(14)

$$
m u^{\prime}+n v^{\prime}+c w^{\prime}=0
$$

$$
(d+m u+n v+c w) u^{\prime}=-\frac{m p^{\prime}}{\rho}+v\left(m^{2}+n^{2}+c^{2}\right) u^{\prime \prime}
$$




$$
\begin{gathered}
(d+m u+n v+c w) v^{\prime}=-\frac{n p^{\prime}}{\rho}+v\left(m^{2}+n^{2}+c^{2}\right) v^{\prime \prime} \\
(d+m u+n v+c w) w^{\prime}=-\frac{c p^{\prime}}{\rho}+v\left(m^{2}+n^{2}+c^{2}\right) w^{\prime \prime}
\end{gathered}
$$

On integrating the Eq. (15) yields

$$
m u+n v+c w=\varepsilon_{0}
$$

After eliminating pressure $p$ from Eqs. (16)-(18) and making use of Eq. (19), we have

$$
\begin{gathered}
v\left(m^{2}+n^{2}+c^{2}\right) u^{\prime \prime}-\left(d+\varepsilon_{0}\right) u^{\prime}=0 \\
v\left(m^{2}+n^{2}+c^{2}\right) v^{\prime \prime}-\left(d+\varepsilon_{0}\right) v^{\prime}=0 \\
v\left(m^{2}+n^{2}+c^{2}\right) w^{\prime \prime}-\left(d+\varepsilon_{0}\right) w^{\prime}=0 \\
u^{\prime \prime}-A u^{\prime}=0
\end{gathered}
$$

The solution of Eq. (23) is

$$
u=\varepsilon_{1}+\varepsilon_{2} e^{A \xi}
$$

where $A=\frac{\left(d+\varepsilon_{0}\right)}{v\left(m^{2}+n^{2}+c^{2}\right)}$. Similarly the solution of $v$, and $w$ components of the momentum equation.

$$
\begin{aligned}
& v=\varepsilon_{3}+\varepsilon_{4} e^{A \xi} \\
& w=\varepsilon_{5}+\varepsilon_{6} e^{A \xi}
\end{aligned}
$$

where $\varepsilon_{0}, \varepsilon_{1}, \varepsilon_{2}, \varepsilon_{3}, \varepsilon_{4}, \varepsilon_{5}$, and $\varepsilon_{6}$ are constants of integration. For Newtonain applicaton solution can be written in original variables.

$$
\begin{aligned}
& u(x, y, z, t)=\varepsilon_{1}+\varepsilon_{2} e^{A(m x+n y+c z+d t)} \\
& v(x, y, z, t)=\varepsilon_{3}+\varepsilon_{4} e^{A(m x+n y+c z+d t)} \\
& w(x, y, z, t)=\varepsilon_{5}+\varepsilon_{6} e^{A(m x+n y+c z+d t)}
\end{aligned}
$$

provided

$$
m \varepsilon_{2}+n \varepsilon_{4}+c \varepsilon_{6}=0
$$

\section{Application $2(3+1$ couple stress fluid)}

With the growing importance of the fluids in contemporary technology Stokes [21] formulated the theory of the couple stress fluid. One of the application of couple stress fluid is its use of mechanisms of lubrications of synovial joints, which has become the object of scientific research. The mathematical setting of the three dimensional couple stress fluid is

$$
\begin{gathered}
\nabla \cdot V=0 \\
\rho\left(V_{t}+(V . \nabla) V\right)=-\nabla p+\left(\mu-\eta \nabla^{2}\right) \nabla^{2} V
\end{gathered}
$$

By taking $V=(u(x, y, z, t), v(x, y, z, t), w(x, y, z, t)), p=$ $p(x, y, z, t)$ flow equations are given as

$$
u_{x}+v_{y}+w_{z}=0
$$

$$
\begin{aligned}
& u_{t}+u u_{x}+v u_{y}+w u_{z}=-\frac{p_{x}}{\rho}+v\left(u_{x x}+u_{y y}+u_{z z}\right) \\
& -\eta^{*}\left(\begin{array}{c}
u_{x x x x}+u_{y y y y}+u_{z z z z} \\
+2\left(u_{x x y y}+u_{y y z z}+u_{x x z z}\right)
\end{array}\right) \\
& v_{t}+u v_{x}+v v_{y}+w v_{z}=-\frac{p_{y}}{\rho}+v\left(v_{x x}+v_{y y}+v_{z z}\right) \\
& -\eta^{*}\left(\begin{array}{c}
v_{x x x x}+v_{y y y y}+v_{z z z z} \\
+2\left(v_{x x y y}+v_{y y z z}+v_{x x z z}\right)
\end{array}\right)
\end{aligned}
$$

$$
\begin{aligned}
& w_{t}+u w_{x}+v w_{y}+w w_{z}=-\frac{p_{z}}{\rho}+v\left(w_{x x}+w_{y y}+w_{z z}\right) \\
& -\eta^{\star}\left(\begin{array}{c}
w_{x x x x}+w_{y y y y}+w_{z z z z} \\
+2\left(w_{x x y y}+w_{y y z z}+w_{x x z z}\right)
\end{array}\right)
\end{aligned}
$$

The methodology discussed in the previous application is also followed in this model. The system (33)-(36) has been reduced to ordinary differential equations

$$
m u^{\prime}+n v^{\prime}+c w^{\prime}=0
$$

$$
\begin{aligned}
u^{\prime}(d+m u+n v+c w)= & -\frac{m p^{\prime}}{\rho}+v\left(m^{2}+n^{2}+c^{2}\right) u^{\prime \prime} \\
& -\eta^{\star} u^{i v}\left(m^{2}+n^{2}+c^{2}\right)^{2}
\end{aligned}
$$




$$
\begin{aligned}
v^{\prime}(d+m u+n v+c w)= & -\frac{n p^{\prime}}{\rho}+v\left(m^{2}+n^{2}+c^{2}\right) v^{\prime \prime} \\
& -\eta^{*} v^{i v}\left(m^{2}+n^{2}+c^{2}\right)^{2} \\
w^{\prime}(d+m u+n v+c w)= & -\frac{c p^{\prime}}{\rho}+v\left(m^{2}+n^{2}+c^{2}\right) w^{\prime \prime} \\
& -\eta^{\star} w^{i v}\left(m^{2}+n^{2}+c^{2}\right)^{2}
\end{aligned}
$$

On integrating the Eq. (37) yields

$$
m u+n v+c w=a_{0}
$$

On eliminating pressure from the system of equations (38)-(40), and substituting Eq. (41) gives

$\left(d+a_{0}\right) u^{\prime}=v\left(m^{2}+n^{2}+c^{2}\right) u^{\prime \prime}-\eta^{\star}\left(m^{2}+n^{2}+c^{2}\right)^{2} u^{i v}$

$$
\left(d+a_{0}\right) v^{\prime}=v\left(m^{2}+n^{2}+c^{2}\right) v^{\prime \prime}-\eta^{\star}\left(m^{2}+n^{2}+c^{2}\right)^{2} v^{i v}
$$

$\left(d+a_{0}\right) w^{\prime}=v\left(m^{2}+n^{2}+c^{2}\right) w^{\prime \prime}-\eta^{\star}\left(m^{2}+n^{2}+c^{2}\right)^{2} w^{i v}$

Here two cases will be considered.

I- $d+a_{0}=0$

II- $d+a_{0} \neq 0$

\section{Case -I}

For this case system of the Eqs. (42)-(44) take the form

$$
\begin{aligned}
& u^{i v}-\chi u^{\prime \prime}=0 \\
& v^{i v}-\chi v^{\prime \prime}=0 \\
& w^{i v}-\chi w^{\prime \prime}=0
\end{aligned}
$$

where, $\chi=\frac{v}{\eta^{*}\left(m^{2}+n^{2}+c^{2}\right)}$

On solving give,

$$
\begin{gathered}
u=a_{1}+a_{2} \xi+a_{3} \cosh \left(\sqrt{\chi} \xi+a_{4}\right) \\
v=a_{5}+a_{6} \xi+a_{7} \cosh \left(\sqrt{\chi} \xi+a_{8}\right) \\
w=a_{9}+a_{10} \xi+a_{11} \cosh \left(\sqrt{\chi} \xi+a_{12}\right)
\end{gathered}
$$

Here, $a_{j}, j=0,1,2 \ldots, 12$ are constants of integration. For the case I, solutions in original variables are given

$$
\begin{aligned}
u= & a_{1}+a_{2}(m x+n y+c t+d) \\
& +a_{3} \cos h\left(\sqrt{\chi}(m x+n y+c z+d t)+a_{4}\right)
\end{aligned}
$$

$$
\begin{aligned}
v= & a_{5}+a_{6}(m x+n y+c t+d) \\
& +a_{7} \cosh \left(\sqrt{\chi}(m x+n y+c z+d t)+a_{8}\right) \\
w= & a_{9}+a_{10}(m x+n y+c t+d) \\
+ & a_{11} \cosh \left(\sqrt{\chi}(m x+n y+c z+d t)+a_{12}\right)
\end{aligned}
$$

provided

$$
m a_{2}+n a_{6}+c a_{10}=0, \quad m a_{3}+n a_{7}+c a_{11}=0
$$

In order to validate exact solution, a boundary value problem has been discussed. Taking $w=0, m=-m$, $n=1, c=0, d=0, \chi=-\frac{\varepsilon_{0}}{2 \eta^{*}}$. In this case plane flow is considered, which led the solutions depend on both $x$ and $y$ coordinates. An exponential type exact solution is found for the example. The boundary conditions are given by

$$
\begin{gathered}
u(0, y)=U_{0}\left(1-e^{-\lambda y}\right), \quad u_{y}(x, \infty)=0, \\
v(x, \infty)=v_{0}, \quad v_{y}(x, \infty)=0, \\
\Omega(x, 0)=0, \quad \Omega(x, \infty)=0
\end{gathered}
$$

Invoking the boundary conditions (55) into Eqs. (45)-(46), we get

$$
u(x, y)=U_{0}\left(1-e^{-\sqrt{\chi}(y-m x)}\right)
$$

$$
v(x, y)=v_{0}-m U_{0} e^{-\sqrt{x}(y-m x)}
$$

and $\Omega=v_{x}-u_{y}$ is the vorticity. Note that $a_{3}, a_{4}, a_{5}, a_{6}$ are related to couple stress fluid. Since $a_{3}=a_{5}=0$ equations (45)-(46), satisfying the Newtonian solution. For $m=0$ solution (56) reduces to that given by [20] and $\chi$ is selected as positive. The effects of couple stress parameter on the fluid velocity components $u, v$ are illustrated in Figures $1-2$ for the $\varepsilon_{0}=-0.1$. Fig. 1 depicts that variation in the velocity of component $u$ interm of couple stress parameter. It is seen that $u$ is the decreasing function of $\eta^{\star}$. Thus it is observed that an increase in the couple stress parameter has a decreasing influence on the velocity $u$. It is also evident from Fig. 2 that the behavior of the couple stress parameter on the $v$ is opposite to that of $u$.

As a second example, we consider the mud flow over a porous surface. The porosity and the suction velocity escalates over the length. Naturally, a mud flow, that occurred specially on the slopes surrounding young, narrow and asymmetric depression basins, may spread on detrial porous sediments starting less porous sandy parts to other gravely parts of the plain. The velocity profile is linear in $y$. The boundary conditions for this case can be written as follows:

$u(x, 0)=0, u(-\infty, y)=u_{0} y, v(x, 0)=v_{0} x, v_{y}(x, \infty)=0$, 


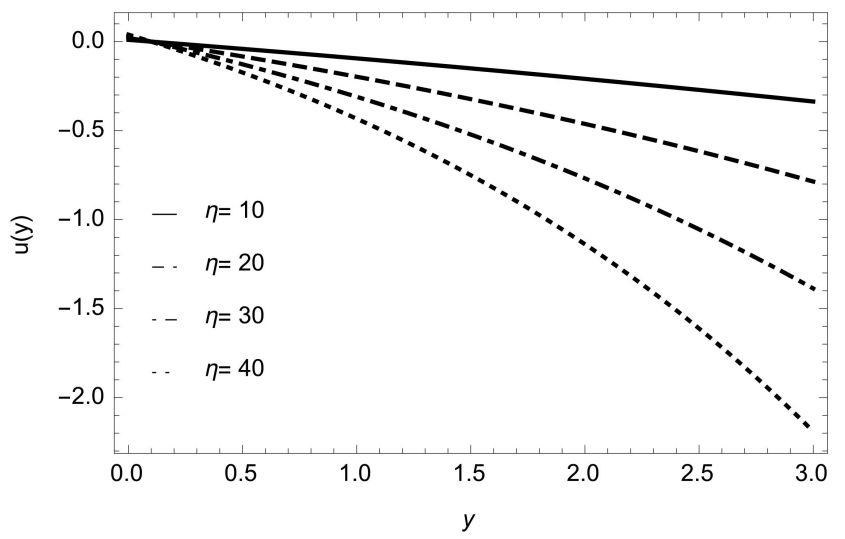

Fig. 1. Effect of couple stress for horizontal component of velocity versus $y\left(m=1, u_{0}=v_{0}=2\right)$.

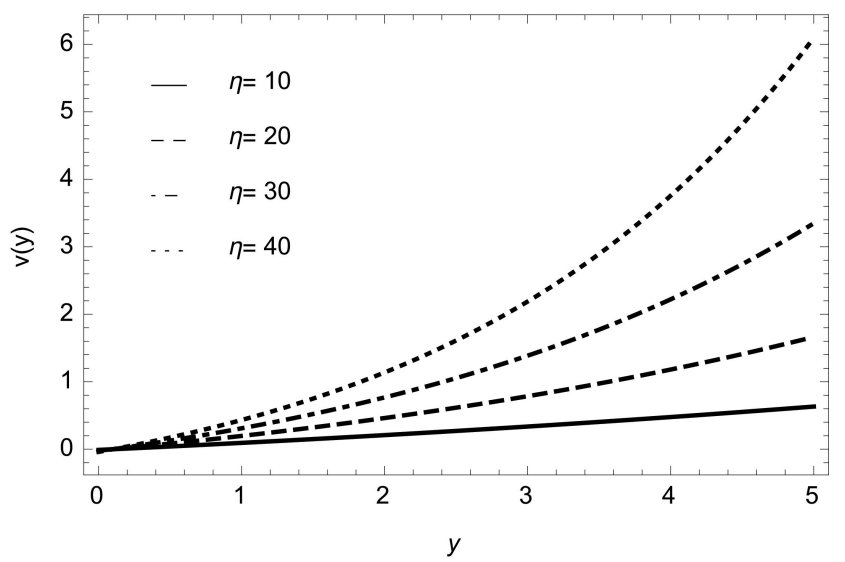

Fig. 2. Effect of couple stress for vertical component of velocity versus $y\left(m=1, U_{0}=v_{0}=2\right)$.

$$
\Omega(x, 0)=0, \quad \Omega(x, \infty)=0
$$

Applying the boundary conditions to Eqs. (45)-(46), we obtain

$$
\begin{gathered}
u(x, y)=u_{0} y \\
v(x, y)=-v_{0} x
\end{gathered}
$$

It is worth mentioning that constants associated with couple stress fluid are vanished under these boundary conditions. This solution satisfying the Newtonian flow.

\section{Case -II}

In this case system of Eqs. (42)-(44) take the form

$$
\begin{gathered}
u^{i v}-\chi u^{\prime \prime}+\delta u^{\prime}=0 \\
v^{i v}-\chi v^{\prime \prime}+\delta v^{\prime}=0 \\
w^{i v}-\chi w^{\prime \prime}+\delta w^{\prime}=0
\end{gathered}
$$

where, $\chi=\frac{v}{\eta^{*}\left(m^{2}+n^{2}+c^{2}\right)}$, and $\delta=\frac{\left(d+a_{0}\right)}{\eta^{*}\left(m^{2}+n^{2}+c^{2}\right)^{2}}$ which is on integration give,

$$
\begin{aligned}
& u=a_{13}+a_{14} e^{\lambda_{1} \xi}+a_{15} e^{\lambda_{2} \xi}+a_{16} e^{\lambda_{3} \xi} \\
& v=a_{17}+a_{18} e^{\lambda_{1} \xi}+a_{19} e^{\lambda_{2} \xi}+a_{20} e^{\lambda_{3} \xi} \\
& w=a_{21}+a_{22} e^{\lambda_{1} \xi}+a_{23} e^{\lambda_{2} \xi}+a_{24} e^{\lambda_{3} \xi}
\end{aligned}
$$

where $\lambda_{i}$ are the roots of equation

$$
\lambda^{3}-\chi \lambda+\delta=0
$$

where $a_{j}$, are constants of integration. The equation for this case in original variables take the form.

$$
\begin{aligned}
u= & a_{13}+a_{14} e^{\lambda_{1}(m x+n y+c z+d t)}+a_{15} e^{\lambda_{2}(m x+n y+c z+d t)} \\
& +a_{16} e^{\lambda_{3}(m x+n y+c z+d t)} \\
v= & a_{17}+a_{18} e^{\lambda_{1}(m x+n y+c z+d t)}+a_{19} e^{\lambda_{2}(m x+n y+c z+d t)} \\
& +a_{20} e^{\lambda_{3}(m x+n y+c z+d t)} \\
w= & a_{21}+a_{22} e^{\lambda_{1}(m x+n y+c z+d t)}+a_{23} e^{\lambda_{2}(m x+n y+c z+d t)} \\
& +a_{24} e^{\lambda_{3}(m x+n y+c z+d t)}
\end{aligned}
$$

provided

$$
\begin{array}{ll}
a_{14}+a_{18}+a_{22}=0, & a_{15}+a_{19}+a_{23}=0, \\
a_{16}+a_{20}+a_{24}=0, &
\end{array}
$$

It is worth noting that th exponential solution of Eq. (67) exist, depending on the values of the physical parameters involved. Since, square roots may give complex values. if $\chi>0$, taking $\rho>0$ then all solutions are real and imaginary, so complex solution exists for this case. Having chosen the appropriate value of parameter i.e. $\lambda>1.8$ gives the real roots for the case considered. Hence, exponential type solution can be obtained for the set of Eqs. (64)-(67).

\section{Concluding remarks}

In this paper, we have looked at two applications of fluid models in $(3+1)$ dimensions, Newtonain and couple stress fluid respectively. An attempt has been made to obtain an exact solutions of the problems, by using the wave transformation. A methodology is developed, that not only encompasses the exact solutions that have been appeared in the previous publications [14]. Also, the exact solutions of $(3+1)$ system that do not seem to have been reported before. Finally, a set of examples of boundary conditions for the case-I is discussed. The following results can be drawn. 
- Inertial terms (nonlinear terms) have been disappeared automatically when the methodology is applied.

- If couple stress parameter $\eta \rightarrow 0$ in Eq. (32) is reduced to Eq. (10).

- For case-I. It is concluded that velocity profiles strongly depend upon couple stress parameter.

- It is observed that velocity component $u$ is the decreasing function of couple stress parameter.

- It is seen that velocity component $v$ is the increasing function of couple stress parameter.

- For case-II. The equation admits the exponential type of real solution, when $\lambda>1.8$.

\section{References}

[1] Adesanya, S. O., 2012. MHD couple stress pulsatile flow between parallel plates, Z. Naturforsch 67, 647.

[2] Ariman, T. T., Sylvester, N. D., 1973. Microcontinuum fluid mechanics - a review. International Journal of Engineering Science 11, 905-30.

[3] Ariman, T. T., Sylvester, N. D., 1974. Applications of microcontinuum fluid mechanics International Journal of Engineering Science 12, 273-87.

[4] El-Wakil, S. A., Abdou, M .A., 2008. New exact traveling wave solutions of two non-linear physical models. Non-linear analysis Theory Methods and Applications 68, 235-245.

[5] El-Dabe, N. T. M., Hassan, A. A., Mohamed, Mona, A. .A., 2003. Effect of couple stresses on pulsatile hydromagnetic Poiseuille flow. Zeitschrift fur Naturforschung 58a, 204-210.

[6] Fetecau, C., Fetecau, C., 2005. Starting solutions for some unsteady unidirectional flows of second grade flow. International journal of Engineering science 43, 781-789.

[7] Farooq, M., Rahim, M. T., Islam, S., Siddiqui, A. M., 2013. steady Poiseuille flow and heat transfer of couple stress fluid between two parallel plates with variable viscosity. Journal of the association of Arab universities for Basic and Applied sciences http://dx.doi.org/10.1016/j.jaubas.2013.01.004

[8] Ghosh, A. K., Sana, P., 2009. On hyderodynamic flow of oldroyd-B fluid near a pulsating plate. Acta astronautica 64, 272-280.

[9] Hayat, T., Mohyuddin, M. R., Asghar, S., 2005. Some inverse solutions for unsteadian fluid. Tamsui Oxford Journal of Mathematical Sciences 21 (11), 1-20.
[10] Kolani, P. N., Huschilt, K., 1984, Semi-inverse solutions of a non-Newtonian Fluid. International Journal of Non-Linear Mechanics 19 (4), 373-381.

[11] Khan, N. A., Ara, A., Jamil, M., 2009. Traveling waves solution of a micropolar fluid. International Journal of Nonlinear Science $\&$ Numerical Simulation 10, 1121-1125.

[12] Khan, N. A., Mahmood, A., Jamil, M., Khan, N. U., 2010. Traveling wave solutions for MHD Aligned flow of a second grade fluid. International Journal of Chemical Reactor Engineering 8, A163.

[13] Khan, N. A., A. Ara, A., Jamil, M., Yildirim, A., 2011. Traveling wave solutions for MHD Aligned flow of a second grade fluid: A symmetry independent approach. Journal King Saud University Science 24, 63-67.

[14] Khan, N. A., Khan, H., 2014. Exact solution MHD flow of couple stress fluid with heat transfer. Journal of Egyption Mathematical Society. 10.1016/j.joems.2014.10.003.

[15] Labropulu, F., 2000. Exact solutions of non-Newtonian fluid flows with prescribed vorticity. Acta Mechanica 141, (1-2), 1120.

[16] Mohyuddin, M.R., Hayat, T., Asghar, S., Mahomed, F.M., Siddiqui, A.M., 2004. On solutions of som non-linear differential equations arising in Newtonian and non-Newtonian fluids. Nonlinear Dynamics 35, (2), 229-248.

[17] Mohyuddin, M. R., Hayat, T., Asghar, S., Siddiqui, A.M., 2006. On semi-inverse solutions for the time-dependent flows of a second grade fluid. Article ID 54295.

[18] Ramzan, M., Farooq, Alsaedi, M. A., and Hayat, T., 2013. MHD three-dimensional flow of couple stress fluid with Newtonian heating. The European Physical Journal Plus 128, 49.

[19] Rani, H. P., Reddy, G. J., Kim, C. N., 2011. couple stress fluid over an infinite vertical cylinder. Engineering Applied Compututional Fluid Mechanics. 5, 159.

[20] Rajagopal, K. R., 1984. On the creeping flow of the second grade fluids. Journal of non-Newtonian Fluid Mechanics. 15, 239.

[21] Stokes, V. K., 1966. Couple-stresses in fluids. Phys Fluid. 9,1709-15.

[22] Srivastava, L, M., 1986. Peristaltic transport of a couple-stress fluid. Rheologica Acta 25, 638-41.

[23] Shehawey, E. F. E., Mekheimer, K. S., 1994. Couple-stresses in peristaltic transport of fluids Journal of Physics D: Applied Physics 27, 1163-70.

[24] Srinivasacharya, D., Kaladhar, K., 2011. Analytical solution of MHD free convective flow of couple stress fluid in an annulus with Hall and Ion-slip effects. Nonlinear Analysis: Modelling and Control 16 (4), 477-487. 